\title{
Nature teach us how to design new biosensors
}

\author{
Luigi Campanella* \\ Luigi Campanella, Department of chemistry, Sapienza University of Rome
}

Received: November 30,2016; Accepted: December 6,2016; Published: December 20,2016

*Corresponding author: Luigi Campanella, Sapienza University of Rome, Chemistry Dept; Email: luigi.campanella@uniroma1.it

\section{Latter to Editorial}

In our times under the pressure from the increasing public awareness of problems caused by the circulation of toxic compounds in the environment, several products have been reconsidered, also from the point of view of possible danger to man and their polluting effect on the environment.

The production and widespread use of hydrocarbons has attracted the attention of researchers, who have classified them among the most toxic substances. In particular, the US Environmental Protection Agency has classified aromatic hydrocarbons like benzene, toluene, the xylenes, etc, as highly toxic substances. Benzene, for instance, has been identified as cancerogenic agent that can induce acute myeloid leukaemia and other types of lymphoma in $\mathrm{man}^{3}$. Furthermore, these compounds were found to be included among the most widespread polluting substances found not only in the atmosphere but also in groundwater and soil; these three types of pollution coexist and are closely correlated. So that this compound has been added to the list of gaseous pollutants to be monitored in air, especially in cities, because of its presence in green fuel and in work environment, also as a result of the accumulating evidence of its carcinogenic properties.

The standard reference method used for hydrocarbon analysis is gas chromatography.

Mass spectrometry is suitable for identifying even low levels of hydrocarbons, although it is normally too expensive to use for routine analyses. Another available technique is IR analysis; finally, chemiluminescence is also sometimes used for aromatic hydrocarbon analysis.

Researchers of Singapore University published the results of their work to develop a biosensor for benzene determination that satisfied the requirements of speed, cheapness, handiness and transportability and could be used as an alternative to the abovementioned analytical methods, often more rigorous but certainly more complex, costly and difficult to be used for in situ analyses.

The above researchers used whole cells of Pseudomonas putida, which were immobilized by filtering the culture medium through a cellulose acetate membrane. Our research group at Sapienza University of Rome developed an original method for immobilizing whole cells on an agarized culture medium which was successfully used to produce toxicity biosensors based on immobilized yeast cells. The results confirm the possibility of assembling biosensors for the determination of benzene and other aromatic hydrocarbons using suitably immobilized colonies of Pseudomonas putida; the immobilization method used has a strong influence on the biosensor's analytical performances, especially linearity range, selectivity and response time; however, these last two characteristics probably also depend on the procedure used, during the growth phase, to select the cells and boost the growth of the Pseudomonas colony so as to be capable of biodegrading the benzene; the capacity to metabolize benzene or other aromatic hydrocarbons apparently depends on the presence of specific protein subunits forming part of the benzene dioxygenase enzyme contained in the cells of Pseudomonas putida used.

The interaction between biological system and environment has been always studied aiming at the evaluation of the effects caused by organic natural and anthropogenic substances on different ecosystems. These evaluations allow on one side to individuate natural markers to be used for the bio monitoring of the environment and on the other side to study biochemical reactions occurring in the living organisms (target organs). Humans according to well known bibliography can partially metabolise benzene to Trans, trans-muconic acid, so able to act as biomarker of benzene. So a new biosensor to determine benzene based on human kidney tissue as this organ has an enzymatic content (dioxygenase) able to degrade benzene by the same way as Pseudomonas was designed is designed and tested. I recall this story just to focus on a very well known truth to researchers: nature is the best teacher we have, able to give us suggestions, advices, stimuli. We have not to forget.

\section{References}

1. H.L.A. Sacarello, in "The Comprehensive Handbook of Hazardous materials", Lewis Publishers, CNR press, Inc, Boca Raton, 1994.

2. N. Irving Sax, R.J. Lewis, Sr. in "Dangerous Properties of Industrial Materials", Van Nostrand Reinhold, NewYork, 1989. doi:10.1002/ ep. 670100308

3. "Chemical Safety Data Sheets", Vol. 1 "Solvents", Royal Society of Chemistry, Information Services, 1989 
4. S.E. Manahan, in "Environmental Chemistry", Fourth edition, PWS Publishers, Boston, 1984

5. Hai-Meng Tan, Shuan-Pei Cheong and Thiam-Chye Tan: Biosensor and Bioelectronics, 9, 1-10, 1994

6. L. Campanella, G. Crescentini, M.G. D'Onofrio, G. Favero, M. Tomassetti, Ann. Chim., 1996;86:527-538,
7. M. Zamanian and J.R. Mason, Biochem. Journ, 1987;244(3): 611-616. doi:10.1042/bj2440611

8. D.V. Parke, R.T. Williams, Biochemistry, 1952; 54:231-238.

9. D.V. Parke, R.T. Williams, Biochemistry, 1953;55(20):337-340,

10. Y. Kojima, N. Itada, O. Hayaishi, J. Biol: Chem, 1961;236:2223-2228, 